also critical in the care of patients with respiratory disorders or unbalanced $\mathrm{pH}$ and that these disorders cannot be evaluated with the use of pulse oximetry. In particular, a normal pulse oximetry measurement does not exclude important abnormalities in the partial pressure of arterial carbon dioxide $\left(\mathrm{PaCO}_{2}\right)$ or $\mathrm{pH}$. Therefore, serious elevations in the $\mathrm{PaCO}_{2}$, which are indicative of ventilatory dysfunction, can be present when the results of pulse oximetry are normal. ${ }^{2}$ Although noninvasive, transcutaneous $\mathrm{CO}_{2}$ monitoring can be used to complement pulse oximetry, current technologies still require further development and are not indicative of acid-base status. ${ }^{3}$ Thus, clinicians need to be aware that levels of arterial and capillary blood gases remain a critical component of the evaluation of gas exchange.

\section{H.S. Jeffrey Man, M.D.}

Philip A. Marsden, M.D.

University of Toronto

Toronto, ON, Canada

p.marsden@utoronto.ca

No potential conflict of interest relevant to this letter was reported.

1. Case Records of the Massachusetts General Hospital (Case 7-2011). N Engl J Med 2011;364:957-66. [Errata, N Engl J Med 2011;364:1281, 1682.]

2. Curley G, Laffey JG, Kavanagh BP. Bench-to-bedside review: carbon dioxide. Crit Care 2010;14:220.

3. Tobias JD. Transcutaneous carbon dioxide monitoring in infants and children. Paediatr Anaesth 2009;19:434-44.

THE AUTHORS AND A COLLEAGUE REPLY: Avidan and Levin point out that the determination of hemoglobin concentrations for pulse oximetry are approximations. In the formulas shown in the video, we could have indicated that hemoglobin var- iables are estimates of the underlying true concentrations by inserting a tilde or a caret above the abbreviation for hemoglobin ( $\hat{\mathrm{H} b})$, but we made the decision to use a simplified approach to clarify the teaching point, as is done in many textbooks.

Even the reference measurement of $\mathrm{SaO}_{2}$ in arterial blood performed by our institution's blood gas laboratory involves the estimation of the relative concentrations of oxyhemoglobin and deoxyhemoglobin in an arterial blood sample with the use of infrared absorption co-oximetry. One can argue that all measurements are estimates and that the question of whether a particular monitoring technique constitutes a measurement or an estimate becomes semantic.

Regarding the inability of pulse oximetry to detect hypercarbia and metabolic disturbances, Man and Marsden bring up important points. Pulse oximetry is an excellent oxygenation monitor, but it does not alert the clinician to changes in ventilation in a timely manner, particularly when supplemental oxygen is used. Anesthesiologists are well aware of this limitation of pulse oximetry and recommend the use of capnography to monitor ventilation during procedural sedation.

Rafael Ortega, M.D.

Christopher Connor, M.D., Ph.D.

\section{Boston Medical Center}

Boston, MA

\section{Kelly Elterman, M.D.}

Brigham and Women's Hospital

Boston, MA

Since publication of their article, the authors report no further potential conflict of interest.

\title{
Case 10-2011: Fever, Confusion, and Liver Failure
}

TO THE EDITOR: With regard to the Case Record of a 60-year-old woman with fever, confusion, and liver failure (March 31 issue) ${ }^{1}$ : we are interested in Tierney's thoughts on the possibility that the combined use of pegylated interferon and ribavirin to treat the patient's hepatitis $\mathrm{C}$ induced an autoimmune liver disease that contributed to her death. ${ }^{2,3}$ Her confusion and asterixis (suggesting hepatic encephalopathy), the markedly elevated levels of transaminases and the associated positive test result for autoimmune markers, and the response to glucocorticoids lead us to suspect a diagnosis of autoimmune liver disease. We are also curious as to whether the preexistence of cirrhosis affects the decision to include splenomegaly in the diagnostic criteria for hemophagocytic lymphohistiocytosis.

\footnotetext{
Christopher Kandel, M.D.

Gabor Kandel, M.D.

University of Toronto

Toronto, ON, Canada

christopher.kandel@utoronto.ca
} 
No potential conflict of interest relevant to this letter was reported.

1. Case Records of the Massachusetts General Hospital (Case 10-2011). N Engl J Med 2011;364:1259-70.

2. Ghany MG, Strader DB, Thomas DL, Seeff LB. Diagnosis, management, and treatment of hepatitis C: an update. Hepatology 2009;49:1335-74.

3. García-Buey L, García-Monzón C, Rodriguez S, et al. Latent autoimmune hepatitis triggered during interferon therapy in patients with chronic hepatitis C. Gastroenterology 1995;108:17707.

TO THE EDITOR: Tierney et al. present the case of a woman with hemophagocytic lymphohistiocytosis who is treated with immunosuppressive therapy. Unfortunately, after an initial improvement, severe infectious complications developed that led to her death. Hemophagocytic syndrome is caused by the uncontrolled activation of macrophages and the release of proinflammatory cytokines (a cytokine storm). However, this activation concerns the macrophage-derived proinflammatory cytokines of the interleukin-1 family (interleukin$1 \alpha$ and $1 \beta$ and interleukin-18), whereas levels of T-cell-derived cytokines, such as interferon- $\gamma$, are much less increased. ${ }^{1}$ Classic immunosuppressive agents indiscriminately inhibit all components of host defense, leading to severe complications, but a more refined strategy has been used successfully in the treatment of hemophagocytic syndrome. Inhibition of the effects of interleukin-1 by its receptor antagonist (interleukin-1Ra, or anakinra) dampens systemic inflammation while leaving untouched host defense mechanisms such as specific cellular immunity. ${ }^{2-4}$ Anakinra has also been used successfully and with few infectious complications in the treatment of other autoinflammatory conditions related to hemophagocytic syndrome. ${ }^{5}$ Therefore, anakinra might be considered as an alternative therapy, with less severe complications, in patients with hemophagocytic syndrome.

Mihai G. Netea, M.D., Ph.D.

Marcel van Deuren, M.D., Ph.D.

Jos W.M. van der Meer, M.D., Ph.D.

Radboud University Nijmegen Medical Center Nijmegen, the Netherlands

m.netea@aig.umcn.nl

No potential conflict of interest relevant to this letter was reported.

1. Sumegi J, Barnes MG, Nestheide SV, et al. Gene expression profiling of peripheral blood mononuclear cells from children with active hemophagocytic lymphohistiocytosis. Blood 2011; 117(15):e151-e160.

2. Behrens $E M$, Kreiger PA, Cherian S, Cron RQ. Interleukin 1 receptor antagonist to treat cytophagic histiocytic panniculitis with secondary hemophagocytic lymphohistiocytosis. J Rheumatol 2006;33:2081-4.

3. Miettunen PM, Jayanthan A, Narendran A. Successful use of anakinra, a soluble IL-1 receptor antagonist, in pediatric rheumatic diseases associated with macrophage activation syndrome/reactive hemophagocytic lymphohistiocytosis. Pediatr Rheumatol 2008;6:Suppl 1:S13.

4. Lurati A, Teruzzi B, Salmaso A, et al. Macrophage activation syndrome (MAS) during anti-IL-1 receptor therapy (anakinra) in a patient affected by systemic onset idiopathic juvenile arthritis (so-JIA): a report and review of the literature. Pediatr Rheumatol Online J 2005;3:79-85. (http://www.pedrheumonlinejournal.org/ mar-apr\%2005/pdf/case\%20report\%20-\%20mas.pdf.)

5. Dinarello CA. Interleukin-1 in the pathogenesis and treatment of inflammatory diseases. Blood 2011;117:3720-32.

TO THE EDITOR: The presentation of the second patient in this case, a young man with fatal hemophagocytic lymphohistiocytosis and active infection with the Epstein-Barr virus, is reminiscent of X-linked lymphoproliferative disease, or Duncan's syndrome (Online Mendelian Inheritance in Man [OMIM] number, 308240), which is caused by mutations in $S H 2 D 1 A,{ }^{1}$ or of Xlinked familial hemophagocytic lymphohistiocytosis (OMIM number, 300635), caused by BIRC4 mutations. ${ }^{2,3}$ In the presence of SH2D1A mutations, serum amyloid P (SAP) component is truncated or absent, which disrupts its capacity to bind with the family of receptors known as SLAM (signaling lymphocytic activation molecule), which in turn disrupts the related signal transduction pathways. The mechanism through which BIRC4 mutations result in the disease phenotype remains unknown. Patients may be asymptomatic until the occurrence of a disease-initiating event, such as infection with Epstein-Barr virus. Patients harboring SH2D1A or BIRC4 mutations may present with hemophagocytic lymphohistiocytosis or dysgammaglobulinemia in association with the virus, but malignant lymphoma develops only in those with SH2D1A mutations. ${ }^{4}$ If this young man's DNA is available, it should be tested for SH2D1A and BIRC4 mutations (given informed consent from a member of the immediate family).

\section{Claudio Sandoval, M.D. \\ New York Medical College \\ Valhalla, NY \\ claudio_sandoval@nymc.edu}

No potential conflict of interest relevant to this letter was reported.

1. Coffey AJ, Brooksbank RA, Brandau O, et al. Host response to $\mathrm{EBV}$ infection in $\mathrm{X}$-linked lymphoproliferative disease results from mutations in an SH2-domain encoding gene. Nat Genet 1998;20:129-35. 
2. Rigaud S, Fondanèche MC, Lambert N, et al. XIAP deficiency in humans causes an X-linked lymphoproliferative syndrome. Nature 2006;444:110-4.

3. Marsh RA, Madden L, Kitchen BJ, et al. XIAP deficiency: a unique primary immunodeficiency best classified as X-linked familial hemophagocytic lymphohistiocytosis and not as X-linked lymphoproliferative disease. Blood 2010;116:1079-82.

4. Sumegi J, Johnson J, Filipovich A, et al. Lymphoproliferative disease, X-linked. In: Pajon RA, Bird TD, Dolan CR, Stephens K, eds. GeneReviews. Seattle: University of Washington, 1993-2011. (http://www.ncbi.nlm.nih.gov/books/NBK1116.)

THE DISCUSSANTS REPLY: The suggestion from Kandel and Kandel that therapy for hepatitis $\mathrm{C}$ may have worsened the first patient's clinical condition by inducing autoimmune hepatitis is entirely plausible, since interferon-ribavirin treatment is associated with autoimmune phenomena, such as autoimmune hemolytic anemia, in other body systems. Given that the precipitants of hemophagocytic lymphohistiocytosis in adults are for the most part not understood, however, and given the rarity of the condition, it is possible that what was observed simply represented the natural history of this serious disorder. Indeed, Tierney had cared for a patient with a clinical course nearly identical to that of this patient before these drugs were developed. Many factors may be responsible for initiating the hyperfunction of macrophages; this common end point makes it difficult to identify the precipitating cause.
Netea et al. propose an elegant therapeutic alternative in anakinra, which offers the advantage of highly selective suppression of immune function and may minimize toxicity. The effects of treatment with anakinra are difficult to study since the number of adult patients with hemophagocytic lymphohistiocytosis is so small. The greater incidence in children, especially in Asia, which is quite often associated with Epstein-Barr virus infection, might make this fertile ground for such investigation, but it cannot be assumed that the pathogenesis in the pediatric and adult populations is similar. It is not clear whether the use of anakinra is applicable to both children and adults, but surely the idea is sound.

Sandoval suggests that the second patient mentioned in the case should be tested for mutations in SH2DIA and BIRC4, which may be associated with fatal infection with Epstein-Barr virus in young men. Genetic testing was performed before this patient died, and no mutations were found.

Lawrence Tierney, M.D.

VA Medical Center

San Francisco, CA

Amy C. Sievers, M.D.

Dana-Farber Cancer Institute

Boston, MA

Since publication of their article, the authors report no further potential conflict of interest.

\section{Eosinophil Granule Protein Localization in Eosinophilic Endomyocardial Disease}

TO THE EDITOR: A 70-year-old man with chronic renal disease, splenomegaly, and eosinophilia presented with transient weakness in the left arm, visual disturbances, disequilibrium, and anomia. Magnetic resonance imaging of the brain showed findings consistent with numerous, small embolic strokes. Four echocardiograms, including a transesophageal echocardiogram, did not reveal abnormalities. Therapeutic trials of glucocorticoids and imatinib were unsuccessful at reducing the numbers of peripheral-blood eosinophils. To investigate the possibility that the emboli were the sequelae of eosinophilic endomyocardial disease, the patient underwent a biopsy of his right ventricle and intraventricular septum. Staining of the myocardium with hematoxylin and eosin did not show clinically significant abnormalities. Notably, only rare eosinophils were detected in the interstitium (Fig. 1A and 1B). However, immunohistologic examination with fluorescein-conjugated antibodies to eosinophil granule major basic protein 1 (MBP1) revealed striking extracellular deposits of eosinophil granule MBP1 (Fig. 1C and 1D). The eosinophil granule MBP1 was most prominent at apparent luminal surfaces, especially over an area of endocardium that stained most intensely.

Eosinophilic endomyocardial disease is a complication of the hypereosinophilic syndrome. A previous study showed eosinophil infiltration and deposition of toxic eosinophil granule proteins in damaged cardiac tissues. ${ }^{2}$ Eosinophil granule 